Let $n=5$; then $q=1$. An arbitrary function of the determinant

$$
\triangle \equiv\left|\begin{array}{rrrrrr}
0 & 1 & 1 & 1 & 1 & 1 \\
1 & 0 & 12 & 13 & 14 & 15 \\
1 & 21 & 0 & 23 & 24 & 25 \\
1 & 31 & 32 & 0 & 34 & 35 \\
1 & 41 & 42 & 43 & 0 & 45 \\
1 & 51 & 52 & 53 & 54 & 0
\end{array}\right|
$$

where $12,13, \cdots$, have the signification given by the identity and equation (2), is a general solution of the simultaneous system (3) for $n=5$. In particular the vanishing of $\Delta$ satisfies the system (3) and hence expresses the relation among the mutual distances of five points in space, a result known to Lagrange. The fifth order determinant $\Delta_{0}$, the minor of $\Delta$ with regard to the upper left hand corner element, equated to zero expresses the necessary and sufficient condition that five points be on a sphere. Similarly the vanishing of $\Delta_{00}$ and that of $\Delta_{000}$ give the conditions, respectively, that four points be coplanar and three points collinear.

Construct the determinant $\Delta$ for $n$ points and call it $D$. $D=0$ is then a generalization of the theorem of Lagrange expressed by $\Delta=0$. This extension is warranted by the form of (3), the symmetry of $\Delta$, and the fact that the invariants considered are absolute invariants.

BALTIMORE,

March 25, 1897.

NOTE ON THE FUNDAMENTAL THEOREMS OF LIE'S THEORY OF CONTINUOUS GROUPS.

BY DR. EDGAR ODELL LOVETT.

(Read before the American Mathematical Society at the Meeting of October $30,1897$.

Lie's theory of continuous groups rests upon the following three fundamental theorems:*

* See LIE: Vorlesungen über continuierliche Gruppen, herausgegeben von Scheffers, Leipzig, 1893, chapter XV; LIE: Theorie der Transformationsgruppen, bearbeitet unter Mitwirkung von Engel, Leipzig, Erster Abschnitt, 1888, chapters II, IV, IX, XVII; zweiter Abschnitt, 1890, chapter XVII; dritter Abschnitt, 1893, chapter XXV. 
I. If the equations

$$
x_{i}^{\prime}=f_{i}\left(x_{1}, \cdots, x_{n}, a_{1}, \cdots, a_{r}\right), \quad i=1, \cdots, n
$$

determine $\infty^{r}$ different transformations which form a group, then there exist $r \quad n$ equations of the form

$\frac{\partial x_{i}^{\prime}}{\partial a_{k}}=\sum_{i}^{r} j \psi_{j k}\left(a_{1}, \cdots, a_{r}\right) \xi_{j i}\left(x_{1}{ }^{\prime}, \cdots, x_{n}{ }^{\prime}\right) \quad(i=1, \cdots, n, k=1, \cdots, r)(\beta)$

and also their solutions

$\xi_{j i}\left(x_{1}{ }^{\prime}, \cdots, x_{n}{ }^{\prime}\right)=\sum_{1}^{r} k \alpha_{j k}\left(a_{1}, \cdots, a_{r}\right) \frac{\partial x_{i}^{\prime}}{\partial a_{k}},(i=1, \cdots, n, j=1, \cdots, r)(\gamma)$

while the $\xi_{j i}\left(x^{\prime}\right)$ do not satisfy $n$ linear homogeneous relations

$$
e_{1} \xi_{1 i}\left(x^{\prime}\right)+\cdots+e_{r} \xi_{r i}\left(x^{\prime}\right) \equiv 0 \quad(i=1, \cdots, n)
$$

with constant coefficients $e_{1}, \cdots, e_{r}$.

Conversely, if $n$ equations $(\alpha)$ which represent $\infty^{r}$ different transformations, satisfy $r \quad n$ equations of the form $(\beta)$ and in consequence of these also $r$ equations of the form $(\gamma)$, if in addition for certain constant quantities $a_{1}^{0}, \cdots, a_{r}^{0}$ there exist the equations

$$
f_{i}\left(x_{1}, \cdots, x_{n}, a_{1}^{0}, \cdots, a_{r}^{0}\right) \equiv x_{i} \quad(i=1, \cdots, n)
$$

and finally the determinant of the $\alpha_{j k}\left(a^{0}\right)$ is different from zero and infinity, the equations $(\alpha)$ represent a group.

II. $r$ independent infinitesimal transformations

$$
X_{i} f \equiv \sum_{1}^{n} \nu \xi_{i \nu}\left(x_{1}, \cdots, x_{n}\right) \frac{\partial f}{\partial x_{\nu}} \quad(i=1, \cdots, r)
$$

generate an $r$ parameter group in the case when the $X_{1} f, \cdots, X_{r} f$, taken two by two, satisfy relations of the form

$$
\left(X_{i} X_{k}\right) \equiv \sum_{1}^{r} s c_{i k s} X_{s} f \quad(i, k=1, \cdots, r)
$$

with constant coefficients $c_{i k s}$, and in no other case. This group contains the identical transformation and its transformations are inverse in pairs.

III. $r^{3}$ constants $c_{i k s}$ determine the structure of an $r$ parameter group in the case when they satisfy the relations 


$$
\begin{gathered}
c_{i k l}+c_{k i l}=0, \\
\sum_{1}^{r} s\left(c_{i k s} c_{s l t}+c_{k l s} c_{s i t}+c_{l i s} c_{s k t}\right)=0, \quad(i, k, l, t=1, \ldots, r)
\end{gathered}
$$

and in no other.

The converse theorems of theorems II and III are also true.

In his first investigations relative to finite continuous transformation groups, Lie attempted to prove that every $r$ parameter group contains the identical transformation as well as $r$ independent infinitesimal transformations and that it is generated by the latter.* $\mathrm{He}$ soon recognized, however, that in his proof certain implied assumptions had been made relative to the nature of the functions involved; accordingly he confined himself to those groups whose transformations may be arranged in pairs as inverse transformations and showed that the theorem is true for such groups. $\dagger$

Later Engel was able to construct in 1884 a finite continuous group which does not contain the identical transformation and whose transformations are not inverse in pairs, namely the group

$$
x_{1}=a x(\bmod a<1) .
$$

Finally Lie found that the equations of every finite continuous group with $r$ parameters can be derived, by introducing new parameters, and by " analytische Fortsetzung," from the equations of an $r$ parameter group which contains the identical transformation and $r$ independent infinitesimal transformations, while its finite transformations arrange themselves in pairs as inverse.:

It is the object of this note to call attention to a misapprehension, if not an error, in an interesting paper "On a law of combination of operators bearing on the theory of continuous transformation groups," by J. E. Campbell, read before the London Mathematical Society, March 11, 1897, and published in the Proceedings of that body, vol. xxviii., Nos. 593-601, pp. 381-390.

Mr. Campbell takes a statement of the converse theorem $\$$ of theorem II and formulates it as follows: If $x_{1}^{\prime}, \cdots, x_{n}{ }^{\prime}$ is a point obtained from the point $x_{1}, \cdots, x_{n}$ by the operation

\footnotetext{
* See Theorie der Transformationsgruppen, vol. 1, p. 165 ; Archiv for Math. og Naturvid., vol. 1, Christiania, 1876.

† Math. Ann., vol. 16, pp. 441, et seq.

$\ddagger$ Theorie der Transformationsgruppen, vol. 1, p. 163, theorem 26.

\& Theorie der Transformationsgruppen, vol. 1, p. 158.
} 


$$
1+X+\frac{X^{2}}{2 !}+\cdots
$$

and $x_{1}{ }^{\prime \prime}, \cdots, x_{n}{ }^{\prime \prime}$ is a point obtained from the point $x_{1}{ }^{\prime}, \cdots, x_{n}{ }^{\prime}$ by the operation

where

$$
1+Y^{\prime}+\frac{Y^{\prime 2}}{2 !}+\cdots
$$

and

$$
X \equiv \lambda_{1} X_{1}+\cdots+\lambda_{r} X_{r}
$$

$$
Y \equiv \mu_{1} X_{1}+\cdots+\mu_{r} X_{r},
$$

$X_{k}$ denoting the linear operator

$$
\sum_{i=1}^{i=n} \xi_{k i}\left(x_{1}, \cdots, x_{n}\right) \frac{\partial}{\partial x_{i}}
$$

then $x_{1}^{\prime \prime}, \cdots, x_{n}^{\prime \prime}$ can be directly derived from the point $x_{1}, \cdots, x_{n}$ by the operation

where

$$
1+Z+\frac{Z^{2}}{2 !}+\cdots
$$

$$
Z \equiv \nu_{1} X_{1}+\cdots+\nu_{r} X_{r},
$$

provided that, for all values of $k, j$,

$$
X_{k} X_{j}-X_{j} X_{k} \equiv \sum_{s=1}^{s=r} c_{k j s} X_{s}
$$

In Lie's theorem the $\lambda, \mu, \nu$, and $c$ are all constants; Mr. Campbell proposes to prove that the same result holds if they are any functions of the variables. The first step in the proof makes use of Lie's theorem that any transformation of the group may be obtained through repeated operations with an infinitesimal transformation of the group; but by theorem II this is the case only when the $c_{i k s}$ are constants and the infinitesimal transformations independent; accordingly Mr. Campbell's theorem cannot be regarded as established.

The constants $c_{i k s}$ which are here in question play a vital rôle in Lie's theory. They are said to determine the structure of a group. In his method for the resolution of algebraic equations which admit of a given substitution group, Galois referred the problem to the resolution of a series of auxiliary equations of which the number and nature depend 
on the structure of the substitution group. Similarly Lie makes the integration of a system of partial differential equations which admits of a finite continuous group of transformations depend upon the integration of a series of auxiliary systems, and the number of these systems, their nature and the way in which they are related to one another depends on the structure of the continuous group. The importance of the structure of finite continuous groups is further illustrated in Picard's theory of linear differential equations and more generally in the theory of differential equations which admit of a fundamental system of integrals.

Princeton, N. J.,

1 October, 1897.

\section{A GEOMETRICAL LOCUS CONNECTED WITH A SYSTEM OF COAXIAL CIRCLES.}

BY PROFESSOR THOMAS F. HOLGATE.

(Read before the American Mathematical Society at its Fourth Summer Meeting, Toronto, Canada, August 16, 1897.)

Suppose there be given a sheaf or pencil of rays whose centre is $P$ and a system of coaxial circles lying in the same plane. Two rays of the sheaf will be tangent to each circle of the system and two circles of the system will be touched by each ray of the sheaf. If we start with any one circle of the system $k_{1}$ and one of its tangent rays $m_{1}$, it is easy to determine the second circle $k_{2}$ to which this ray is tangent, then the second ray $m_{2}$ tangent to $k_{2}$, then the second circle $k_{3}$ to which $m_{2}$ is tangent and so on indefinitely, the circles and rays forming a continuous chain. Whether or not this chain of circles and rays will return into itself depends upon the location of the centre $P$ with respect to the system of coaxial circles and I undertake in the present paper to find the locus of the point $P$ for which the chain of circles and rays will close with three circles and three rays.

In other words, I undertake to find the locus of points through which three lines can be drawn tangent to three circles of a coaxial system in pairs.

Any two circles and a pair of common tangents form such a closed circuit containing two elements of each kind and the point $P$ may be any one of the six vertices of the complete quadrilateral formed by the four tangents common to the two chosen circles. 